\title{
The Transitive Closures of Matrices over Idempotent Semirings and its Applications
}

\author{
Wang Zhixi ${ }^{1}$, Wang Yana ${ }^{1}$, He Yong ${ }^{1}$, Hu Binliang ${ }^{2}$ \\ ${ }^{1}$ School of Computer Science and Engineering, Hunan University of Science and Technology, Xiangtan 411201, Hunan, China \\ ${ }^{2}$ Colleges of Electromechanical Engineering, Hunan University of Science and Technology, Xiangtan 411201, Hunan, China \\ zhixiwang@163.com, wxyhnust@163.com, ynghe@263.net, blhu@hnust.edu.cn
}

\begin{abstract}
The Algebraic Path Problem is an important issue with a broad background. Consider the problem to solve the algebraic path problem can be concluded to find the Kleene closure of the adjacency matrix, an algorithm of time complexity $O\left(n^{3}\right)$ to find the transitive closure of matrices over idempotent semiring is constructed, as well as the conditions applicable to it are provided. Compared with the Gauß-Jordan elimination, this algorithm could extend the applicable range to certain semirings which do not have completeness and closeness, Thus, it has a wide application since it can provide a new method to solve the algebraic path problem over idempotent semirings which do not have completeness and closeness.
\end{abstract}

Index Terms - Idempotent semiring, Transitive Closures, Transitive_Closure Algorithm, Algebraic Path Problem.

\section{Introduction}

The Algebraic Path Problem, which is to perform a special unary operation, called closure, on a square matrix over semirings based on semirings-algebraic theory, is an important issue with a broad background. This problem generalizes some matrix problems(such as computing the inverse of a real-valued matrix), graph problems(e.g. transitive closure, shortest path, the stochastic communication network problem), and regular language problems(e.g. the proof of the correspondence between regular expressions and finite automata). Algebraic path problem and its algorithms have a wide application in the field of network engineering, image processing and pattern recognition.

In 1956, Kleene proved that every regular language can be represented by a regular expression. Actually, it was the first algorithm for the algebraic path problem[1]. And in 1962, Warshall algorithm for computing the transitive closure and Floyd algorithm for shortest path problem were respectively proposed[2-3]. Later, Gaussian elimination for computing the inverse of matrices was also presented[4-5]. Warshall algorithms, Floyd algorithms and Gaussian elimination method are classical algorithms for these three problems respectively [2-4]. Until 1971, Carré discovered the similarities between these algorithms and proposed their general form Gauß-Jordan elimination for solving them based on semirings algebra theory. The algorithm for solving algebraic path problem boiled down to find the Kleene closure of adjacency matrix, i.e., the summation of all nonnegative integer powers of the adjacency matrix[6]. Since then, with an in-depth study, Gauß-Jordan elimination method has been improved from every angle and its application has a gradual expansion from some special semirings first to general cases, and the computing power with the introduction of advanced technologies such as parallel computing has been greatly improved [7-14]. However, the semirings applied to Gau $\beta$ - Jordan elimination method always required completeness and closeness.

Note that the Kleene Closures of matrices over Idempotent semirings are easily obtained by their Transitive Closures. This paper constructs an algorithm to find the Transitive Closures of matrices over idempotent semiring and provides conditions applicable to it. Actually, the algorithm extends the applicable range of Gau $\beta$-Jordan elimination method to certain semirings which do not have completeness and closeness.

\section{Preliminary}

Let $R$ be a non-empty set defined the addition $\oplus$ and the multiplication $\otimes$. If $(R, \oplus)$ is a commutative monoid, and $(R, \otimes)$ is a semigroup, moreover, multiplication $\otimes$ satisfies the distributive law to addition $\oplus$, then the algebraic system $R=(R, \oplus, \otimes)$ is called a semiring. Usually, the product $a \otimes b$ of elements $a, b$ over semiring $R$ is denoted by $a b$. Denote that $a^{(m)}=a \oplus a^{2} \oplus \ldots \oplus a^{m}$ for the element $a$ of $R$ and positive integer $m$.

Assume that $R$ is a semiring. If $\overline{1} \in R$ is the identity element of $(R, \otimes)$, then we say that $\overline{1}$ is the identity element of $R$. If $\overline{0} \in R$ is the identity element of $(R, \oplus)$ and the zero element of $(R, \otimes)$, then we say $\overline{0}$ is the zero element of $R$.

Define the relation $\leq$ over semiring $R$ as $a \leq b \Leftrightarrow a \oplus b=a$. We call $R$ an idempotent semiring if for any $a \in R$, then $a \leq a$. It is easy to prove that the relation $\leq$ over the idempotent semirings is a partial order relation. The idempotent semirings have a significant application in solving optimization problems and the classification of regular languages, c.f. [14-16].

For the element $a$ of $R$, let $\Omega(a)=\{x \in R \mid x \leq a, x \leq x x\}$, we call that $a^{t}$ is the transitive closure of $a$ if $a^{t} \in \Omega(a)$ and for any $x \in \Omega(A), x \leq a^{t}$.

\footnotetext{
*This work is supported by National Nature Science Foundation of China (No:61272196), Hunan Provincial Natural Science Foundation of China ( No: 10JJ3067), and Scientific Research Fund of Hunan Provincial Education Department (No: 11C0534).
} 
Similar to the numerical matrices, we can define matrices and their addition and multiplication over semiring $R$. Denote the element $(i, j)-$ of $A$ over $R$ by $A_{i j}$, and matrix that the element $(i, j)-$ is $a_{i j}$ over $R$ by $\left(a_{i j}\right)$. The set of all $n$-square matrix over $R$ is recorded as $M_{n}(R)$. Then $M_{n}(R)$ with regard to matrix addition and multiplication also forms a semiring called an $n$-square matrix semiring over $R$. The relation $\leq$ on $M_{n}(R)$ is defined as: $A \leq B \Leftrightarrow A_{i j} \leq B_{i j}$ for $1 \leq i, j \leq n$. Obviously, $M_{n}(R)$ is idempotent if and only if $R$ is idempotent. For the $n$-square matrix $A$ over $R$ and positive integer $m$, we set $A^{(m)}=A \oplus A^{2} \oplus \ldots \oplus A^{m}$, and denote the element $(i, j)-$ of $A^{m}$ by $A_{i j}^{m}$, and also the element $(i, j)$ - of $A^{(m)}$ by $A_{i j}^{(m)}$. The basic properties of matrices over semirings and the details of semirings can be found in literature [15].

For the $n$-square matrix $A$ over $R$ and the nonnegative integer $k$ with $k \leq n$, we define matrices $A^{[k]}=\left(A_{i j}^{[k]}\right)$ recursively as follows:

$$
A_{i j}^{[k]}= \begin{cases}A_{i j}, & k=0 \\ A_{i j}^{[k-1]}, & i=k \text { or } j=k \\ A_{i j}^{k-1]} \oplus A_{i k}^{[k-1]} A_{k j}^{[k-1]}, & 1 \leq k \leq n \text { and } i \neq k \text { and } j \neq k\end{cases}
$$

\section{The Analysis Of The Complexity Of Transitive_Closure Algorithm}

The following theorem is the main conclusion of this article, and we will consider the proof of this matter in next section.

Theorem 1. Let $R$ is an idempotent semiring, $A \in M_{n}(R)$, then $A^{t}=A^{[n]}$ if and only if $A_{i j}^{[n]} \leq A_{i k}^{[n]} A_{k j}^{[n]}$ for $1 \leq i, j, k \leq n$.

Theorem 1 provides an algorithm to compute the transitive closures of the $n$-square matrix over idempotent semiring, the algorithm can be described by the pseudo codes as follows:

\section{Transitive Closure Algorithm}

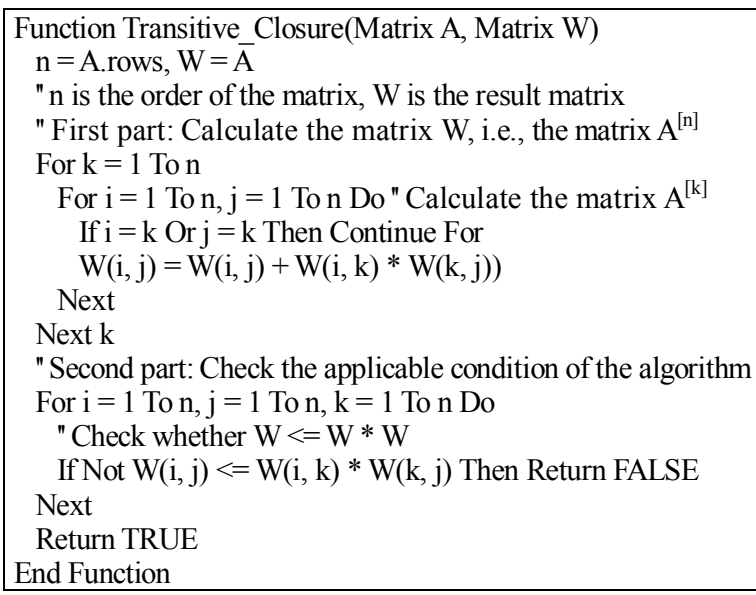

\section{The Analysis of The Complexity of Algorithm.}

The algorithm firstly is to compute the matrices $A^{[n]}$, then is to check the applicable conditions of the algorithm. Taken the assignment, addition, multiplication and comparison operations of the elements of the semiring as a basic operation respectively, we can conclude that the first part needs $3 n(n-1)^{2}$ times of basic operations, and the second part takes up at most $2 n^{3}$ times of the basic operations. Hence, the time complexity of the algorithm is $O\left(n^{3}\right)$, which has the same order with the time complexity of the Floyd algorithm and the Gauß-Jordan algorithm, c.f. [4], [12].

\section{Proof Of The Transitive_Closure Algorithm}

In this section, we assume that $R$ is an idempotent semiring, $n$ is a given positive integer, and $A=\left(a_{i j}\right) \in M_{n}(R)$.

We need Lemma 2 to Lemma 4 as follows to prove the Transitive_Closure algorithm, i.e., the Theorem 1.

Lemma 2 Let $a, b, c, d, x_{i} \in R$ with $1 \leq i \leq m$. Then the following condition holds:

(1) $a \leq \sum_{i=1}^{m} x_{i}$ if and only if $a \leq x_{i}$, for $i=1,2, \ldots, m$.

(2) If there exists an integer $i$ such that $x_{i} \leq a$, then $\sum_{i=1}^{m} x_{i} \leq a$.

(3) If $a \leq b, c \leq d$, then $a c \leq b d$.

Proof. (1) Assume that $a \leq x_{i}$ with $i=1,2, \ldots, m$, then $a \oplus \sum_{i=1}^{m} x_{i}=\sum_{i=1}^{m}\left(a \oplus x_{i}\right)=\sum_{i=1}^{m} a=a$.

Conversely, assume that $a \leq \sum_{i=1}^{m} x_{i}$, then for each $x_{i}$, we have $a \oplus x_{i}=a \oplus \sum_{i=1}^{m} x_{i} \oplus x_{i}=a \oplus \sum_{i=1}^{m} x_{i}=a$.

(2) Assume that there exists an integer $i$ such that $x_{i} \leq a$. Further assume that $i=1$, then

$$
a \oplus \sum_{i=1}^{m} x_{i}=\left(a \oplus x_{1}\right) \oplus \sum_{i=2}^{m} x_{i}=\sum_{i=1}^{m} x_{i} .
$$

(3) Assume that $a \leq b, c \leq d$, then

$$
\begin{aligned}
a c \oplus b d & =(a \oplus b)(c \oplus d) \oplus b d \\
& =a c \oplus a d \oplus b c \oplus b d \oplus b d \\
& =a c \oplus a d \oplus b c \oplus b d \\
& =(a \oplus b)(c \oplus d) \\
& =a c
\end{aligned} .
$$

Lemma 3. Assume $a \in R, x \in \Omega(a)$, then the following conditions holds:

(1) $a^{(r)} \oplus a^{(p)} a^{(q)}=a^{(\max (r, p+q))}$, where $r, p, q$ are all positive integers.

(2) For any positive integer $m, x \leq a^{m}$ and $x \leq a^{(m)}$.

(3) If there exists a positive integer $m$ such that $a^{(m)} \leq x$, then $a^{t}=x \quad\left(=a^{(m)}\right)$. 
Proof. (1) By the idempotence of $M_{n}(R)$, we can conclude that

$$
\begin{aligned}
a^{(r)} \oplus a^{(p)} a^{(q)} & =a^{(r)} \oplus \sum_{i=1}^{p} a^{i} \otimes \sum_{i=1}^{q} a^{i} \\
& =a^{(r)} \oplus \sum_{i=1}^{p} \sum_{j=1}^{q} a^{i+j} \\
& =a^{(r)} \oplus a \oplus \sum_{i=2}^{p+q} a^{i} \\
& =a^{(r)} \oplus a^{(p+q)} \\
& =a^{(\max (r, p+q))}
\end{aligned}
$$

(2) The proof is by mathematical induction. It is evident that $x \leq a$ is trivial. Assume that for some positive integer $m$, $x \leq a^{m}$. From the fact that $w \in \Omega(a)$ and by (3) of Lemma 2, we can easily get that $x \leq x x \leq a a^{m}=a^{m+1}$, thus, for any positive integer $m$, we have $x \leq a^{m}$. Recall that (1) of Lemma 2, we obtain $x \leq a^{(m)}$ for any positive integer $m$.

(3) By applying (2) and the anti-symmetry property, we obtain $x=a^{(m)}$. Moreover, it is also clear that for any $w \in \Omega(A), w \leq a^{(m)}=x$. Then, by the definition of $a^{t}$, we have $a^{t}=x=a^{(m)}$.

Lemma 4. For any positive integer $k$ with $k \leq n$, we have $A^{\left(2^{k}\right)} \leq A^{[k]}$.

Proof. By the definition of $A^{[1]}$ and (2) of Lemma 2, we can get $A^{(2)}=A \oplus A^{2} \leq A^{[1]}$. By induction hypothesis, assume that for some positive integer $k$ with $k<n$, we have $A^{\left(2^{k}\right)} \leq A^{[k]}$, then $A^{\left(2^{k+1}\right)}=A^{\left(2^{k}\right)} \oplus A^{\left(2^{k}\right)} A^{\left(2^{k}\right)} \leq A^{[k]} \oplus A^{[k]} A^{[k]} \leq A^{[k+1]}$ is obtained by the definition of $A^{[k+1]},(2)$ of Lemma 2 and (1) of Lemma 3. For above all, then we have:

Proof of Theorem 1.

By (1) of Lemma 2, we can conclude that for $1 \leq i, j, k \leq n, A_{i j}^{[n]} \leq A_{i k}^{[n]} A_{k j}^{[n]}$ is equivalent to $A^{[n]} \leq A^{[n]} A^{[n]}$.

$\Rightarrow$ ) If $A^{t}=A^{[n]}$, by the definition of $A^{t}, A^{[n]} \leq A^{[n]} A^{[n]}$.

$\Leftarrow)$ Since $A^{[n]} \leq A$ and $A^{[n]} \leq A^{[n]} A^{[n]}$, we can obtain $A^{[n]} \in \Omega(A)$. Then, we also have $A^{\left(2^{n}\right)} \leq A^{[n]}$ by Lemma 4 . and hence by (3) of Lemma 3, $A^{t}=A^{[n]}$.

\section{The Application Of Transitive_Closure Algorithm}

\section{A. The Plus Closure of Matrices}

The sum $a^{+}=a \oplus a^{2} \oplus a^{3}+\cdots$ of all positive integer power of element $a$ in semiring $R$ is called the Plus Closure of $a$. If $R$ is an idempotent semiring and $A \in M_{n}(R)$ satisfies $A^{[n]} \leq A^{[n]} A^{[n]}$, then $A^{[n]} \in \Omega(A)$. By (2) of Lemma 3, we obtain $A^{[n]} \leq A^{+}$. Moreover, Lemma 4 allows to show $A^{+} \leq A^{\left(2^{n}\right)} \leq A^{[n]}$. Consequently, the anti-symmetry property yields that $A^{+}=A^{[n]}$. Therefore, Transitive_Closure algorithm can be used to compute the Plus Closure of matrices over idempotent semirings.

\section{B. Judgment Of Loop_Nonnegative Matrices}

The element $x$ is non-negative if it satisfies the relation $\overline{1} \leq x$ in semiring $R$. For the $n$-square matrix $A=\left(a_{i j}\right)$ over semiring $R$, we call that $A$ is loop non-negative if all of the elements in $\left\{a_{i_{1} i_{2}} a_{i_{2} i_{3}} \ldots a_{i_{m} i_{1}} \mid 1 \leq m, 1 \leq i_{1}, i_{2}, \ldots, i_{m} \leq n\right\}$ are nonnegative.

Theorem 2. Let $R$ is an idempotent semiring, and $A \in M_{n}(R)$. If $A^{[n]} \leq A^{[n]} A^{[n]}$, then $A$ is loop_nonnegative if and only if $\overline{1} \leq A_{i i}^{[n]}$, for $1 \leq i \leq n$.

Proof. Since $A^{[n]} \leq A^{[n]} A^{[n]}$, thus we can easily know $A^{+}=A^{[n]}$. And by (1) of Lemma 2, we also have: $\overline{1} \leq a_{i_{1} i_{2}} a_{i_{2} i_{3}} \ldots a_{i_{m} i_{1}}$ if and only if $\overline{1} \leq A_{i_{1} i_{1}}^{+}=A_{i_{1} i_{1}}^{[n]}$, that is to say, $A$ is loop_nonnegative if and only if for $1 \leq i \leq n, \overline{1} \leq A_{i i}^{[n]}$.

We can conclude by Theorem 2 that Transitive_Closure algorithm can be used to judge whether the $n$-square matrix $A$ over the idempotent semiring $R$ is loop_nonnegative after some appropriate changes. The method is to add the following statements before the statement "Return TRUE", where "Identity" denotes $\overline{1}$.

For $\mathrm{i}=1$ To $n$ Do " Check Identity $<=\mathrm{W}(\mathrm{i}, \mathrm{i})$
If Not Identity $<=\mathrm{W}(\mathrm{i}, \mathrm{i})$ Then Return FALSE "NOT
Next

\section{The Algebraic Path Problems}

Let $R$ be a semiring, $G=(V, E)$ be a (directed) graph, where $V=\{1,2, \cdots, n\}$ is the vertices set, and $E \subseteq V \times V$ is the edges set. The set of all paths from vertex $i$ to vertex $j$ is denoted by $i \rightarrow j$. the length of the path $p$ in $G$ is denoted by $|p|$. For a given function $w: E \rightarrow R$, we can define the weighted graph $G=(V, E, w)$ over $R$. The image $w(e)$ of function $w$ is called the value of $e$. The adjacency matrix $A(G)=\left(w_{i j}\right)$ of $G$ is defined as follows:

$$
w_{i j}= \begin{cases}w((i, j)), & (i, j) \in E \\ 0, & (i, j) \notin E\end{cases}
$$

For $p=\left(p_{0}, p_{1}, \ldots, p_{k}\right) \in i \rightarrow j$, we say that $w(p)=\prod_{i=0}^{k} w\left(p_{i}\right)$ is defined as $d_{i j}=\sum_{p \in i \rightarrow j} w(p)$.

Algebraic path problems over a semiring is actually to find the distance between any two vertices $i$ and $j$ over the weighted graph $G=(V, E, w)$ of $R$. If there exist solutions to the algebraic path problems, we call that the matrix $S(G)=\left(d_{i j}\right)$ is the solution matrix of $G$. Obviously, if $(A(G))^{+}$ exists, then $S(G)=(A(G))^{+}$. Consequently, If $R$ is an idempotent semiring and $A=A(G)$ satisfies the relation $A^{[n]} \leq A^{[n]} A^{[n]}$, Transitive_Closure algorithm is an effective method to find the solution matrix $S(G)$ of $G$. Actually, if the process of computing the Kleene Closure $A^{*}=I_{n}+A^{+}$of 
$A$ can be added to the algorithm, where $I_{n}$ is the $n$-square identity matrix, then we can obtain the same results as the Gauß-Jordan elimination method. In this case, there is no need to require completeness and closeness of $R$.

\section{The Shortest Path Problem Over Totally Ordered Idempotent Semiring}

If $R$ is an idempotent semiring and the partial order relation $\leq$ over $R$ is a total ordering relation, we say that $R$ is a total ordering idempotent semiring. For any two vertices $i, j$ of $G$, note that even if $d_{i j}$ may exists, the shortest path from $i$ to $j$ may still not. When $R$ is a total ordering idempotent semiring and $A=A(G)$ satisfies the relation $A^{[n]} \leq A^{[n]} A^{[n]}$, then by the total ordering property of the partial ordering relation $\leq$ over $R$, necessarily there exists a shortest path from $i$ to $j$. Therefore, Modeled on Floyd algorithms, we can construct an algorithm based on Transitive_Closure algorithm to solve the shortest path problems over $R$.

\section{Acknowledgment}

This work is supported by National Nature Science Foundation of China (No:61272196), Hunan Provincial Natural Science Foundation of China ( No: 10JJ3067), and Scientific Research Fund of Hunan Provincial Education Department (No: 11C0534).

\section{References}

[1] Kleenee S. C. Representation of events in nerve nets and finite automata. Annals Of Mathematics Studies 34, Automata Studies, eds: J. McCarthy and C.E. Shannon, 1956, page 3-41.
[2] Warshall S. A Theorem on Boolean Matrices[J]. Journal of the ACM, 1962, 9(1): 11-12.

[3] Floyd R W. Algorithm 97: Shortest path[J]. Communications of the ACM, 1962, 5(6): 345.

[4] Dasgupta S. Papadimitriou C. Vazirani U. Algorithms[M], The McGrawHill Companies, Inc., New York, 2008.

[5] Forsythe G. E. and Moller C. B. computer solution of linear algebraic systems. Prentice Hall, Englewood Cliffs, NJ,1967.

[6] Carré B A. An algebra for network routine problems[J]. Journal of the Institute of Mathematical and Its Applications, 1971, 7: 273-294.

[7] Zimmermann U. Linear and combinatorial optimization in ordered algebraic structures[J]. Annals of Discrete Mathematics, 1981, 10: 1-380.

[8] Gunter-Roze G. A systolic array algorithm for the algebraic path problem (Shortest path; Matrix inversion) [J]. Computing, 1985, 34: 191-219.

[9] Tsai H, Horng S, Tsai S, et al. Solving an algebraic path problem and some related graph problems on a hyper-bus broadcast network[J]. IEEE Transactions on Parallel and Distributed Systems, 1997, 8 (12): 12261235.

[10] Djamegni C T, Quinton P, Rajopadhye S, et al. Derivation of systolic algorithms for the algebraic path problem by recurrence transformations[J]. Parallel Computing, 2000, 26 (17): 1429-1445.

[11] Takahashi A, Sedukhin S. Parallel Blocked Algorithm for solving the algebraic path problem on a matrix processor[J]. Lecture Notes in Computer Science, 2005, 3726: 786-795.

[12] Lluch-Lafuente A, Montanari A. Quantitative $\mu$-calculus and CTL defined over constraint semirings[J]. Theoretical Computer Science, 2005, 346 (1): 135-160.

[13] Mohri M. Semiring frameworks and algorithms for shortest-distance problems[J]. Journal of Automata, Languages and Combinatorics, 2003, 7 (3): 321-350

[14] Penn G. Efficient transitive closure of sparse matrices over closed semirings[J]. Theoretical Computer Science, 2006, 354 (1): 72 - 81.

[15] Polak L. A classification of rational languages by semilattice-ordered monoids[J]. Archiv der Mathematik, 2004, 3153: 635-647.

[16] Golan J S. Semirings and Their Applications[M]. Kluwer Academic Publishers, Dordrecht, 1999. 